\title{
Measurement of the Absolute Branching Fraction of $D^{0} \rightarrow K^{-} \pi^{+}$
}

B. Aubert, ${ }^{1}$ M. Bona, ${ }^{1}$ D. Boutigny, ${ }^{1}$ Y. Karyotakis, ${ }^{1}$ J. P. Lees, ${ }^{1}$ V. Poireau,${ }^{1}$ X. Prudent,${ }^{1}$ V. Tisserand,,${ }^{1}$ A. Zghiche,${ }^{1}$ J. Garra Tico, ${ }^{2}$ E. Grauges, ${ }^{2}$ L. Lopez, ${ }^{3}$ A. Palano, ${ }^{3}$ G. Eigen, ${ }^{4}$ I. Ofte, ${ }^{4}$ B. Stugu, ${ }^{4}$ L. Sun, ${ }^{4}$ G. S. Abrams, ${ }^{5}$ M. Battaglia, ${ }^{5}$ D. N. Brown, ${ }^{5}$ J. Button-Shafer, ${ }^{5}$ R. N. Cahn, ${ }^{5}$ Y. Groysman, ${ }^{5}$ R. G. Jacobsen, ${ }^{5}$ J. A. Kadyk, ${ }^{5}$ L. T. Kerth, ${ }^{5}$

Yu. G. Kolomensky, ${ }^{5}$ G. Kukartsev, ${ }^{5}$ D. Lopes Pegna, ${ }^{5}$ G. Lynch, ${ }^{5}$ L. M. Mir, ${ }^{5}$ T. J. Orimoto, ${ }^{5}$ M. Pripstein, ${ }^{5}$ N. A. Roe, ${ }^{5}$ M. T. Ronan, ${ }^{5}{ }^{*}$ K. Tackmann, ${ }^{5}$ W. A. Wenzel, ${ }^{5}$ P. del Amo Sanchez,${ }^{6}$ C. M. Hawkes, ${ }^{6}$ A. T. Watson, ${ }^{6}$ T. Held, ${ }^{7}$ H. Koch ${ }^{7}$ B. Lewandowski, ${ }^{7}$ M. Pelizaeus, ${ }^{7}$ T. Schroeder,${ }^{7}$ M. Steinke, ${ }^{7}$ W. N. Cottingham, ${ }^{8}$ D. Walker,${ }^{8}$ D. J. Asgeirsson, ${ }^{9}$ T. Cuhadar-Donszelmann, ${ }^{9}$ B. G. Fulsom, ${ }^{9}$ C. Hearty, ${ }^{9}$ N. S. Knecht, ${ }^{9}$ T. S. Mattison, ${ }^{9}$ J. A. McKenna, ${ }^{9}$ A. Khan, ${ }^{10}$ M. Saleem, ${ }^{10}$ L. Teodorescu, ${ }^{10}$ V. E. Blinov, ${ }^{11}$ A. D. Bukin, ${ }^{11}$ V. P. Druzhinin,,${ }^{11}$ V. B. Golubev, ${ }^{11}$ A. P. Onuchin, ${ }^{11}$ S. I. Serednyakov, ${ }^{11}$ Yu. I. Skovpen, ${ }^{11}$ E. P. Solodov, ${ }^{11}$ K. Yu Todyshev, ${ }^{11}$ M. Bondioli, ${ }^{12}$ S. Curry, ${ }^{12}$ I. Eschrich, ${ }^{12}$ D. Kirkby, ${ }^{12}$ A. J. Lankford,${ }^{12}$ P. Lund,${ }^{12}$ M. Mandelkern, ${ }^{12}$ E. C. Martin, ${ }^{12}$ D. P. Stoker, ${ }^{12}$ S. Abachi, ${ }^{13}$ C. Buchanan, ${ }^{13}$ S. D. Foulkes, ${ }^{14}$ J. W. Gary, ${ }^{14}$ F. Liu, ${ }^{14}$ O. Long, ${ }^{14}$ B. C. Shen, ${ }^{14}$ L. Zhang, ${ }^{14}$ H. P. Paar, ${ }^{15}$ S. Rahatlou, ${ }^{15}$ V. Sharma, ${ }^{15}$

J. W. Berryhill, ${ }^{16}$ C. Campagnari, ${ }^{16}$ A. Cunha, ${ }^{16}$ B. Dahmes,${ }^{16}$ T. M. Hong, ${ }^{16}$ D. Kovalskyi, ${ }^{16}$ J. D. Richman, ${ }^{16}$

T. W. Beck, ${ }^{17}$ A. M. Eisner, ${ }^{17}$ C. J. Flacco, ${ }^{17}$ C. A. Heusch, ${ }^{17}$ J. Kroseberg, ${ }^{17}$ W. S. Lockman, ${ }^{17}$ T. Schalk, ${ }^{17}$

B. A. Schumm, ${ }^{17}$ A. Seiden, ${ }^{17}$ D. C. Williams, ${ }^{17}$ M. G. Wilson, ${ }^{17}$ L. O. Winstrom, ${ }^{17}$ E. Chen,${ }^{18}$ C. H. Cheng, ${ }^{18}$

A. Dvoretskii, ${ }^{18}$ F. Fang, ${ }^{18}$ D. G. Hitlin, ${ }^{18}$ I. Narsky, ${ }^{18}$ T. Piatenko, ${ }^{18}$ F. C. Porter, ${ }^{18}$ G. Mancinelli, ${ }^{19}$ B. T. Meadows, ${ }^{19}$

K. Mishra, ${ }^{19}$ M. D. Sokoloff, ${ }^{19}$ F. Blanc,${ }^{20}$ P. C. Bloom, ${ }^{20}$ S. Chen, ${ }^{20}$ W. T. Ford,${ }^{20}$ J. F. Hirschauer, ${ }^{20}$ A. Kreisel, ${ }^{20}$

M. Nagel, ${ }^{20}$ U. Nauenberg, ${ }^{20}$ A. Olivas,${ }^{20}$ J. G. Smith, ${ }^{20}$ K. A. Ulmer, ${ }^{20}$ S. R. Wagner ${ }^{20}$ J. Zhang, ${ }^{20}$ A. M. Gabareen, ${ }^{21}$ A. Soffer, ${ }^{21}$ W. H. Toki, ${ }^{21}$ R. J. Wilson, ${ }^{21}$ F. Winklmeier, ${ }^{21}$ Q. Zeng, ${ }^{21}$ D. D. Altenburg, ${ }^{22}$ E. Feltresi, ${ }^{22}$ A. Hauke, ${ }^{22}$ H. Jasper, ${ }^{22}$ J. Merkel, ${ }^{22}$ A. Petzold, ${ }^{22}$ B. Spaan, ${ }^{22}$ K. Wacker, ${ }^{22}$ T. Brandt, ${ }^{23}$ V. Klose, ${ }^{23}$ H. M. Lacker, ${ }^{23}$ W. F. Mader, ${ }^{23}$

R. Nogowski, ${ }^{23}$ J. Schubert, ${ }^{23}$ K. R. Schubert, ${ }^{23}$ R. Schwierz, ${ }^{23}$ J. E. Sundermann, ${ }^{23}$ A. Volk, ${ }^{23}$ D. Bernard, ${ }^{24}$

G. R. Bonneaud ${ }^{24}$ E. Latour, ${ }^{24}$ V. Lombardo,${ }^{24}$ Ch. Thiebaux,${ }^{24}$ M. Verderi, ${ }^{24}$ P. J. Clark, ${ }^{25}$ W. Gradl,${ }^{25}$ F. Muheim, ${ }^{25}$

S. Playfer, ${ }^{25}$ A. I. Robertson, ${ }^{25}$ Y. Xie, ${ }^{25}$ M. Andreotti, ${ }^{26}$ D. Bettoni ${ }^{26}$ C. Bozzi,${ }^{26}$ R. Calabrese, ${ }^{26}$ A. Cecchi, ${ }^{26}$ G. Cibinetto, ${ }^{26}$ P. Franchini, ${ }^{26}$ E. Luppi,${ }^{26}$ M. Negrini, ${ }^{26}$ A. Petrella,${ }^{26}$ L. Piemontese, ${ }^{26}$ E. Prencipe,${ }^{26}$ V. Santoro, ${ }^{26}$

F. Anulli, ${ }^{27}$ R. Baldini-Ferroli, ${ }^{27}$ A. Calcaterra, ${ }^{27}$ R. de Sangro, ${ }^{27}$ G. Finocchiaro, ${ }^{27}$ S. Pacetti, ${ }^{27}$ P. Patteri, ${ }^{27}$ I. M. Peruzzi, ${ }^{27, \dagger}$ M. Piccolo, ${ }^{27}$ M. Rama, ${ }^{27}$ A. Zallo, ${ }^{27}$ A. Buzzo, ${ }^{28}$ R. Contri,${ }^{28}$ M. Lo Vetere, ${ }^{28}$ M. M. Macri ${ }^{28}$ M. R. Monge, ${ }^{28}$ S. Passaggio, ${ }^{28}$ C. Patrignani, ${ }^{28}$ E. Robutti, ${ }^{28}$ A. Santroni, ${ }^{28}$ S. Tosi, ${ }^{28}$ K. S. Chaisanguanthum, ${ }^{29}$ M. Morii, ${ }^{29}$ J. Wu, ${ }^{29}$ R. S. Dubitzky, ${ }^{30}$ J. Marks, ${ }^{30}$ S. Schenk, ${ }^{30}$ U. Uwer, ${ }^{30}$ D. J. Bard ${ }^{31}$ P. D. Dauncey, ${ }^{31}$ R. L. Flack, ${ }^{31}$ J. A. Nash, ${ }^{31}$ M. B. Nikolich ${ }^{31}$ W. Panduro Vazquez, ${ }^{31}$ P. K. Behera, ${ }^{32}$ X. Chai,${ }^{32}$ M. J. Charles, ${ }^{32}$ U. Mallik, ${ }^{32}$ N. T. Meyer, ${ }^{32}$ V. Ziegler, ${ }^{32}$ J. Cochran, ${ }^{33}$ H. B. Crawley, ${ }^{33}$ L. Dong, ${ }^{33}$ V. Eyges, ${ }^{33}$ W. T. Meyer, ${ }^{33}$ S. Prell, ${ }^{33}$

E. I. Rosenberg, ${ }^{33}$ A. E. Rubin, ${ }^{33}$ A. V. Gritsan, ${ }^{34}$ Z. J. Guo, ${ }^{34}$ C. K. Lae, ${ }^{34}$ A. G. Denig, ${ }^{35}$ M. Fritsch, ${ }^{35}$ G. Schott, ${ }^{35}$ N. Arnaud, ${ }^{36}$ J. Béquilleux, ${ }^{36}$ M. Davier, ${ }^{36}$ G. Grosdidier, ${ }^{36}$ A. Höcker,${ }^{36}$ V. Lepeltier, ${ }^{36}$ F. Le Diberder,${ }^{36}$ A. M. Lutz, ${ }^{36}$

S. Pruvot, ${ }^{36}$ S. Rodier, ${ }^{36}$ P. Roudeau, ${ }^{36}$ M. H. Schune, ${ }^{36}$ J. Serrano, ${ }^{36}$ V. Sordini, ${ }^{36}$ A. Stocchi, ${ }^{36}$ W. F. Wang, ${ }^{36}$ G. Wormser, ${ }^{36}$ D. J. Lange, ${ }^{37}$ D. M. Wright, ${ }^{37}$ C. A. Chavez, ${ }^{38}$ I. J. Forster, ${ }^{38}$ J. R. Fry, ${ }^{38}$ E. Gabathuler, ${ }^{38}$ R. Gamet, ${ }^{38}$ D. E. Hutchcroft, ${ }^{38}$ D. J. Payne, ${ }^{38}$ K. C. Schofield,${ }^{38}$ C. Touramanis, ${ }^{38}$ A. J. Bevan, ${ }^{39}$ K. A. George, ${ }^{39}$ F. Di Lodovico, ${ }^{39}$

W. Menges,${ }^{39}$ R. Sacco,${ }^{39}$ G. Cowan,${ }^{40}$ H. U. Flaecher, ${ }^{40}$ D. A. Hopkins, ${ }^{40}$ P. S. Jackson, ${ }^{40}$ T. R. McMahon, ${ }^{40}$

F. Salvatore, ${ }^{40}$ A. C. Wren, ${ }^{40}$ D. N. Brown,${ }^{41}$ C. L. Davis, ${ }^{41}$ J. Allison, ${ }^{42}$ N. R. Barlow, ${ }^{42}$ R. J. Barlow,${ }^{42}$ Y. M. Chia, ${ }^{42}$ C. L. Edgar, ${ }^{42}$ G. D. Lafferty, ${ }^{42}$ T. J. West, ${ }^{42}$ J. I. Yi ${ }^{42}$ J. Anderson, ${ }^{43}$ C. Chen,${ }^{43}$ A. Jawahery, ${ }^{43}$ D. A. Roberts, ${ }^{43}$ G. Simi, ${ }^{43}$ J. M. Tuggle, ${ }^{43}$ G. Blaylock,${ }^{44}$ C. Dallapiccola,${ }^{44}$ S. S. Hertzbach, ${ }^{44}$ X. Li,${ }^{44}$ T. B. Moore,${ }^{44}$ E. Salvati, ${ }^{44}$ S. Saremi, ${ }^{44}$ R. Cowan, ${ }^{45}$ P. H. Fisher, ${ }^{45}$ G. Sciolla, ${ }^{45}$ S. J. Sekula, ${ }^{45}$ M. Spitznagel, ${ }^{45}$ F. Taylor,${ }^{45}$ R. K. Yamamoto, ${ }^{45}$ S. E. Mclachlin, ${ }^{46}$ P. M. Patel, ${ }^{46}$ S. H. Robertson, ${ }^{46}$ A. Lazzaro, ${ }^{47}$ F. Palombo, ${ }^{47}$ J. M. Bauer ${ }^{48}$ L. Cremaldi, ${ }^{48}$ V. Eschenburg, ${ }^{48}$ R. Godang,${ }^{48}$

R. Kroeger, ${ }^{48}$ D. A. Sanders, ${ }^{48}$ D. J. Summers, ${ }^{48}$ H. W. Zhao, ${ }^{48}$ S. Brunet, ${ }^{49}$ D. Côté, ${ }^{49}$ M. Simard,${ }^{49}$ P. Taras, ${ }^{49}$ F. B. Viaud, ${ }^{49}$ H. Nicholson, ${ }^{50}$ G. De Nardo, ${ }^{51}$ F. Fabozzi, ${ }^{51, \$}$ L. Lista ${ }^{51}$ D. Monorchio,${ }^{51}$ C. Sciacca,${ }^{51}$ M. A. Baak, ${ }^{52}$ G. Raven, ${ }^{52}$ H. L. Snoek, ${ }^{52}$ C. P. Jessop, ${ }^{53}$ J. M. LoSecco, ${ }^{53}$ G. Benelli, ${ }^{54}$ L. A. Corwin, ${ }^{54}$ K. K. Gan, ${ }^{54}$ K. Honscheid, ${ }^{54}$ D. Hufnagel, ${ }^{54}$ H. Kagan,${ }^{54}$ R. Kass, ${ }^{54}$ J. P. Morris, ${ }^{54}$ A. M. Rahimi, ${ }^{54}$ J. J. Regensburger, ${ }^{54}$ R. Ter-Antonyan, ${ }^{54}$ Q. K. Wong, ${ }^{54}$ N. L. Blount,${ }^{55}$ J. Brau,${ }^{55}$ R. Frey, ${ }^{55}$ O. Igonkina, ${ }^{55}$ J. A. Kolb,${ }^{55}$ M. Lu, ${ }^{55}$ R. Rahmat,${ }^{55}$ N. B. Sinev, ${ }^{55}$

D. Strom, ${ }^{55}$ J. Strube,${ }^{55}$ E. Torrence, ${ }^{55}$ N. Gagliardi,${ }^{56}$ A. Gaz,${ }^{56}$ M. Margoni, ${ }^{56}$ M. Morandin, ${ }^{56}$ A. Pompili, ${ }^{56}$

M. Posocco, ${ }^{56}$ M. Rotondo, ${ }^{56}$ F. Simonetto, ${ }^{56}$ R. Stroili, ${ }^{56}$ C. Voci, ${ }^{56}$ E. Ben-Haim, ${ }^{57}$ H. Briand, ${ }^{57}$ J. Chauveau, ${ }^{57}$ P. David ${ }^{57}$ L. Del Buono,${ }^{57}$ Ch. de la Vaissière, ${ }^{57}$ O. Hamon, ${ }^{57}$ B. L. Hartfiel,${ }^{57}$ Ph. Leruste, ${ }^{57}$ J. Malclès,${ }^{57}$ J. Ocariz,${ }^{57}$ 
A. Perez ${ }^{57}$ L. Gladney,${ }^{58}$ M. Biasini,${ }^{59}$ R. Covarelli, ${ }^{59}$ E. Manoni,${ }^{59}$ C. Angelini, ${ }^{60}$ G. Batignani, ${ }^{60}$ S. Bettarini, ${ }^{60}$ G. Calderini, ${ }^{60}$ M. Carpinelli, ${ }^{60}$ R. Cenci, ${ }^{60}$ A. Cervelli, ${ }^{60}$ F. Forti, ${ }^{60}$ M. A. Giorgi ${ }^{60}$ A. Lusiani, ${ }^{60}$ G. Marchiori, ${ }^{60}$ M. A. Mazur, ${ }^{60}$ M. Morganti, ${ }^{60}$ N. Neri, ${ }^{60}$ E. Paoloni, ${ }^{60}$ G. Rizzo, ${ }^{60}$ J. J. Walsh, ${ }^{60}$ M. Haire, ${ }^{61}$ J. Biesiada ${ }^{62}$ P. Elmer,${ }^{62}$ Y. P. Lau, ${ }^{62}$ C. Lu, ${ }^{62}$ J. Olsen, ${ }^{62}$ A. J. S. Smith ${ }^{62}$ A. V. Telnov,${ }^{62}$ E. Baracchini,${ }^{63}$ F. Bellini, ${ }^{63}$ G. Cavoto, ${ }^{63}$ A. D'Orazio, ${ }^{63}$ D. del Re ${ }^{63}$ E. Di Marco, ${ }^{63}$ R. Faccini, ${ }^{63}$ F. Ferrarotto, ${ }^{63}$ F. Ferroni, ${ }^{63}$ M. Gaspero, ${ }^{63}$ P. D. Jackson, ${ }^{63}$ L. Li Gioi, ${ }^{63}$ M. A. Mazzoni, ${ }^{63}$ S. Morganti, ${ }^{63}$ G. Piredda, ${ }^{63}$ F. Polci, ${ }^{63}$ F. Renga, ${ }^{63}$ C. Voena,${ }^{63}$ M. Ebert, ${ }^{64}$ H. Schröder,${ }^{64}$ R. Waldi, ${ }^{64}$ T. Adye, ${ }^{65}$ G. Castelli, ${ }^{65}$ B. Franek, ${ }^{65}$ E. O. Olaiya, ${ }^{65}$ S. Ricciardi, ${ }^{65}$ W. Roethel, ${ }^{65}$ F. F. Wilson, ${ }^{65}$ R. Aleksan,${ }^{66}$ S. Emery, ${ }^{66}$ M. Escalier, ${ }^{66}$ A. Gaidot, ${ }^{66}$ S. F. Ganzhur, ${ }^{66}$ G. Hamel de Monchenault, ${ }^{66}$ W. Kozanecki, ${ }^{66}$ M. Legendre, ${ }^{66}$ G. Vasseur, ${ }^{66}$ Ch. Yèche, ${ }^{66}$ M. Zito, ${ }^{66}$ X. R. Chen, ${ }^{67}$ H. Liu,${ }^{67}$ W. Park, ${ }^{67}$ M. V. Purohit,${ }^{67}$ J. R. Wilson, ${ }^{67}$ M. T. Allen, ${ }^{68}$ D. Aston, ${ }^{68}$ R. Bartoldus, ${ }^{68}$ P. Bechtle, ${ }^{68}$ N. Berger ${ }^{68}$ R. Claus, ${ }^{68}$ J. P. Coleman, ${ }^{68}$ M. R. Convery, ${ }^{68}$ J. C. Dingfelder, ${ }^{68}$ J. Dorfan, ${ }^{68}$ G. P. Dubois-Felsmann, ${ }^{68}$ D. Dujmic, ${ }^{68}$ W. Dunwoodie ${ }^{68}$ R. C. Field, ${ }^{68}$ T. Glanzman, ${ }^{68}$ S. J. Gowdy, ${ }^{68}$ M. T. Graham, ${ }^{68}$ P. Grenier, ${ }^{68}$ C. Hast ${ }^{68}$ T. Hryn'ova, ${ }^{68}$ W. R. Innes, ${ }^{68}$ M. H. Kelsey, ${ }^{68}$ H. Kim ${ }^{68}$ P. Kim, ${ }^{68}$ D. W. G. S. Leith, ${ }^{68}$ S. Li, ${ }^{68}$ S. Luitz, ${ }^{68}$ V. Luth ${ }^{68}$ H. L. Lynch, ${ }^{68}$ D. B. MacFarlane, ${ }^{68}$ H. Marsiske, ${ }^{68}$ R. Messner ${ }^{68}$ D. R. Muller, ${ }^{68}$ C. P. O' Grady, ${ }^{68}$ A. Perazzo, ${ }^{68}$ M. Perl, ${ }^{68}$ T. Pulliam, ${ }^{68}$ B. N. Ratcliff, ${ }^{68}$ A. Roodman, ${ }^{68}$ A. A. Salnikov, ${ }^{68}$ R. H. Schindler,${ }^{68}$ J. Schwiening, ${ }^{68}$ A. Snyder, ${ }^{68}$ J. Stelzer, ${ }^{68}$ D. Su, ${ }^{68}$ M. K. Sullivan, ${ }^{68}$ K. Suzuki, ${ }^{68}$ S. K. Swain, ${ }^{68}$ J. M. Thompson, ${ }^{68}$ J. Va'vra, ${ }^{68}$ N. van Bakel, ${ }^{68}$ A. P. Wagner, ${ }^{68}$ M. Weaver, ${ }^{68}$ W. J. Wisniewski, ${ }^{68}$ M. Wittgen, ${ }^{68}$ D. H. Wright,,${ }^{68}$ A. K. Yarritu, ${ }^{68}$ K. Yi, ${ }^{68}$ C. C. Young, ${ }^{68}$ P. R. Burchat, ${ }^{69}$ A. J. Edwards, ${ }^{69}$ S. A. Majewski, ${ }^{69}$ B. A. Petersen, ${ }^{69}$ L. Wilden, ${ }^{69}$ S. Ahmed, ${ }^{70}$ M. S. Alam,${ }^{70}$ R. Bula,${ }^{70}$ J. A. Ernst, ${ }^{70}$ V. Jain,${ }^{70}$ B. Pan,${ }^{70}$ M. A. Saeed, ${ }^{70}$ F. R. Wappler, ${ }^{70}$ S. B. Zain, ${ }^{70}$ W. Bugg,${ }^{71}$ M. Krishnamurthy, ${ }^{71}$ S. M. Spanier, ${ }^{71}$ R. Eckmann, ${ }^{72}$ J. L. Ritchie, ${ }^{72}$ A. M. Ruland, ${ }^{72}$ C. J. Schilling, ${ }^{72}$ R. F. Schwitters,${ }^{72}$ J. M. Izen, ${ }^{73}$ X. C. Lou, ${ }^{73}$ S. Ye ${ }^{73}$ F. Bianchi, ${ }^{74}$ F. Gallo, ${ }^{74}$ D. Gamba,${ }^{74}$ M. Pelliccioni, ${ }^{74}$ M. Bomben, ${ }^{75}$ L. Bosisio, ${ }^{75}$ C. Cartaro, ${ }^{75}$ F. Cossutti, ${ }^{75}$ G. Della Ricca,${ }^{75}$ L. Lanceri, ${ }^{75}$ L. Vitale, ${ }^{75}$ V. Azzolini, ${ }^{76}$ N. Lopez-March,${ }^{76}$ F. Martinez-Vidal, ${ }^{76}$ D. A. Milanes,${ }^{76}$ A. Oyanguren, ${ }^{76}$ J. Albert,${ }^{77}$ Sw. Banerjee, ${ }^{77}$ B. Bhuyan,${ }^{77}$ K. Hamano, ${ }^{77}$ R. Kowalewski, ${ }^{77}$ I. M. Nugent, ${ }^{77}$ J. M. Roney, ${ }^{77}$ R. J. Sobie, ${ }^{77}$ J. J. Back, ${ }^{78}$ P. F. Harrison, ${ }^{78}$ T. E. Latham, ${ }^{78}$ G. B. Mohanty, ${ }^{78}$ M. Pappagallo, ${ }^{78,8}$ H. R. Band, ${ }^{79}$ X. Chen, ${ }^{79}$ S. Dasu, ${ }^{79}$ K. T. Flood, ${ }^{79}$ J. J. Hollar, ${ }^{79}$ P. E. Kutter, ${ }^{79}$ Y. Pan ${ }^{79}$ M. Pierini, ${ }^{79}$ R. Prepost,${ }^{79}$ S. L. Wu, ${ }^{79}$ Z. Yu, ${ }^{79}$ and H. Neal ${ }^{80}$

(BABAR Collaboration)

\footnotetext{
${ }^{1}$ Laboratoire de Physique des Particules, IN2P3/CNRS et Université de Savoie, F-74941 Annecy-Le-Vieux, France

${ }^{2}$ Universitat de Barcelona, Facultat de Fisica, Departament ECM, E-08028 Barcelona, Spain

${ }^{3}$ Università di Bari, Dipartimento di Fisica and INFN, I-70126 Bari, Italy

${ }^{4}$ University of Bergen, Institute of Physics, N-5007 Bergen, Norway

${ }^{5}$ Lawrence Berkeley National Laboratory and University of California, Berkeley, California 94720, USA

${ }^{6}$ University of Birmingham, Birmingham, B15 2TT, United Kingdom

${ }^{7}$ Ruhr Universität Bochum, Institut für Experimentalphysik 1, D-44780 Bochum, Germany

${ }^{8}$ University of Bristol, Bristol BS8 1TL, United Kingdom

${ }^{9}$ University of British Columbia, Vancouver, British Columbia, Canada V6T 1Z1

${ }^{10}$ Brunel University, Uxbridge, Middlesex UB8 3PH, United Kingdom

${ }^{11}$ Budker Institute of Nuclear Physics, Novosibirsk 630090, Russia

${ }^{12}$ University of California at Irvine, Irvine, California 92697, USA

${ }^{13}$ University of California at Los Angeles, Los Angeles, California 90024, USA

${ }^{14}$ University of California at Riverside, Riverside, California 92521, USA

${ }^{15}$ University of California at San Diego, La Jolla, California 92093, USA

${ }^{16}$ University of California at Santa Barbara, Santa Barbara, California 93106, USA

${ }^{17}$ University of California at Santa Cruz, Institute for Particle Physics, Santa Cruz, California 95064, USA

${ }^{18}$ California Institute of Technology, Pasadena, California 91125, USA

${ }^{19}$ University of Cincinnati, Cincinnati, Ohio 45221, USA

${ }^{20}$ University of Colorado, Boulder, Colorado 80309, USA

${ }^{21}$ Colorado State University, Fort Collins, Colorado 80523, USA

${ }^{22}$ Universität Dortmund, Institut für Physik, D-44221 Dortmund, Germany

${ }^{23}$ Technische Universität Dresden, Institut für Kern- und Teilchenphysik, D-01062 Dresden, Germany

${ }^{24}$ Laboratoire Leprince-Ringuet, CNRS/IN2P3, Ecole Polytechnique, F-91128 Palaiseau, France

${ }^{25}$ University of Edinburgh, Edinburgh EH9 3JZ, United Kingdom

${ }^{26}$ Università di Ferrara, Dipartimento di Fisica and INFN, I-44100 Ferrara, Italy

${ }^{27}$ Laboratori Nazionali di Frascati dell'INFN, I-00044 Frascati, Italy

${ }^{28}$ Università di Genova, Dipartimento di Fisica and INFN, I-16146 Genova, Italy

${ }^{29}$ Harvard University, Cambridge, Massachusetts 02138, USA
} 
${ }^{30}$ Universität Heidelberg, Physikalisches Institut, Philosophenweg 12, D-69120 Heidelberg, Germany

${ }^{31}$ Imperial College London, London, SW7 2AZ, United Kingdom

${ }^{32}$ University of Iowa, Iowa City, Iowa 52242, USA

${ }^{33}$ Iowa State University, Ames, Iowa 50011-3160, USA

${ }^{34}$ Johns Hopkins University, Baltimore, Maryland 21218, USA

${ }^{35}$ Universität Karlsruhe, Institut für Experimentelle Kernphysik, D-76021 Karlsruhe, Germany

${ }^{36}$ Laboratoire de l'Accélérateur Linéaire, IN2P3/CNRS et Université Paris-Sud 11, Centre Scientifique d'Orsay, B. P. 34, F-91898 ORSAY Cedex, France

${ }^{37}$ Lawrence Livermore National Laboratory, Livermore, California 94550, USA

${ }^{38}$ University of Liverpool, Liverpool L69 7ZE, United Kingdom

${ }^{39}$ Queen Mary, University of London, E1 4NS, United Kingdom

${ }^{40}$ University of London, Royal Holloway and Bedford New College, Egham, Surrey TW20 OEX, United Kingdom

${ }^{41}$ University of Louisville, Louisville, Kentucky 40292, USA

${ }^{42}$ University of Manchester, Manchester M13 9PL, United Kingdom

${ }^{43}$ University of Maryland, College Park, Maryland 20742, USA

${ }^{44}$ University of Massachusetts, Amherst, Massachusetts 01003, USA

${ }^{45}$ Massachusetts Institute of Technology, Laboratory for Nuclear Science, Cambridge, Massachusetts 02139, USA

${ }^{46}$ McGill University, Montréal, Québec, Canada H3A $2 T 8$

${ }^{47}$ Università di Milano, Dipartimento di Fisica and INFN, I-20133 Milano, Italy

${ }^{48}$ University of Mississippi, University, Mississippi 38677, USA

${ }^{49}$ Université de Montréal, Physique des Particules, Montréal, Québec, Canada H3C 3J7

${ }^{50}$ Mount Holyoke College, South Hadley, Massachusetts 01075, USA

${ }^{51}$ Università di Napoli Federico II, Dipartimento di Scienze Fisiche and INFN, I-80126, Napoli, Italy

${ }^{52}$ NIKHEF, National Institute for Nuclear Physics and High Energy Physics, NL-1009 DB Amsterdam, The Netherlands

${ }^{53}$ University of Notre Dame, Notre Dame, Indiana 46556, USA

${ }^{54}$ Ohio State University, Columbus, Ohio 43210, USA

${ }^{55}$ University of Oregon, Eugene, Oregon 97403, USA

${ }^{56}$ Università di Padova, Dipartimento di Fisica and INFN, I-35131 Padova, Italy

${ }^{57}$ Laboratoire de Physique Nucléaire et de Hautes Energies, IN2P3/CNRS, Université Pierre et Marie Curie-Paris6, Université Denis Diderot-Paris7, F-75252 Paris, France

${ }^{58}$ University of Pennsylvania, Philadelphia, Pennsylvania 19104, USA

${ }^{59}$ Università di Perugia, Dipartimento di Fisica and INFN, I-06100 Perugia, Italy

${ }^{60}$ Università di Pisa, Dipartimento di Fisica, Scuola Normale Superiore and INFN, I-56127 Pisa, Italy

${ }^{61}$ Prairie View A\&M University, Prairie View, Texas 77446, USA

${ }^{62}$ Princeton University, Princeton, New Jersey 08544, USA

${ }^{63}$ Università di Roma La Sapienza, Dipartimento di Fisica and INFN, I-00185 Roma, Italy

${ }^{64}$ Universität Rostock, D-18051 Rostock, Germany

${ }^{65}$ Rutherford Appleton Laboratory, Chilton, Didcot, Oxon, OX11 OQX, United Kingdom

${ }^{66}$ DSM/Dapnia, CEA/Saclay, F-91191 Gif-sur-Yvette, France

${ }^{67}$ University of South Carolina, Columbia, South Carolina 29208, USA

${ }^{68}$ Stanford Linear Accelerator Center, Stanford, California 94309, USA

${ }^{69}$ Stanford University, Stanford, California 94305-4060, USA

${ }^{70}$ State University of New York, Albany, New York 12222, USA

${ }^{71}$ University of Tennessee, Knoxville, Tennessee 37996, USA

${ }^{72}$ University of Texas at Austin, Austin, Texas 78712, USA

${ }^{73}$ University of Texas at Dallas, Richardson, Texas 75083, USA

${ }^{74}$ Università di Torino, Dipartimento di Fisica Sperimentale and INFN, I-10125 Torino, Italy

${ }^{75}$ Università di Trieste, Dipartimento di Fisica and INFN, I-34127 Trieste, Italy

${ }^{76}$ IFIC, Universitat de Valencia-CSIC, E-46071 Valencia, Spain

${ }^{77}$ University of Victoria, Victoria, British Columbia, Canada V8W 3P6

${ }^{78}$ Department of Physics, University of Warwick, Coventry CV4 7AL, United Kingdom

${ }^{79}$ University of Wisconsin, Madison, Wisconsin 53706, USA

${ }^{80}$ Yale University, New Haven, Connecticut 06511, USA

(Received 18 April 2007; published 6 February 2008)

We measure the absolute branching fraction for $D^{0} \rightarrow K^{-} \pi^{+}$using partial reconstruction of $\bar{B}^{0} \rightarrow$ $D^{*+} X \ell^{-} \bar{\nu}_{\ell}$ decays, in which only the charged lepton and the pion from the decay $D^{*+} \rightarrow D^{0} \pi^{+}$are used. Based on a data sample of $230 \times 10^{6} B \bar{B}$ pairs collected at the $Y(4 S)$ resonance with the $B A B A R$ detector at the PEP-II asymmetric-energy $B$ factory at SLAC, we obtain $\mathcal{B}\left(D^{0} \rightarrow K^{-} \pi^{+}\right)=(4.007 \pm 0.037 \pm$ $0.072) \%$, where the first uncertainty is statistical and the second is systematic. 
The decay $D^{0} \rightarrow K^{-} \pi^{+}[1]$ is a reference mode for the measurements of the branching fractions of the $D^{0}$ to any other final state. A precise measurement of the value of $\mathcal{B}\left(D^{0} \rightarrow K^{-} \pi^{+}\right)$improves our knowledge of most of the decays of the $B$ mesons, and of fundamental parameters of the Standard Model. For instance, the largest systematic uncertainty on the branching ratio $\mathcal{B}\left(B^{0} \rightarrow D^{*-} \ell^{+} \nu_{\ell}\right)$, and the experimental uncertainty on the determination of the Cabibbo-Kobayashi-Maskawa matrix element $V_{\mathrm{cb}}$ from that semileptonic decay are induced by the uncertainty on $\mathcal{B}\left(D^{0} \rightarrow K^{-} \pi^{+}\right)$.

CLEO- $c$ [2] has recently published the most precise result on this branching fraction, which is widely used [3]. We present here a more precise measurement based on a different technique. We identify $D^{0} \rightarrow K^{-} \pi^{+}$decays in a sample of $D^{0}$ mesons from $D^{*+} \rightarrow D^{0} \pi^{+}$decays and obtained with partial reconstruction of $\bar{B}^{0} \rightarrow D^{*+}(X) \ell^{-} \bar{\nu}_{\ell}$.

The data sample used in this analysis consists of an integrated luminosity of $210 \mathrm{fb}^{-1}$, corresponding to $230 \times$ $10^{6} B \bar{B}$ pairs, collected at the $\Upsilon(4 S)$ resonance (onresonance) and $22 \mathrm{fb}^{-1}$ collected $40 \mathrm{MeV}$ below the resonance (off-resonance) by the $B A B A R$ detector. The offresonance events are used to subtract the non- $B \bar{B}$ (continuum) background. A simulated sample of $B \bar{B}$ events with integrated luminosity equivalent to approximately 5 times the size of the data sample is used for efficiency computation and background studies.

A detailed description of the BABAR detector is provided elsewhere [4]. High-momentum particles are reconstructed by matching hits in the silicon vertex tracker (SVT) with track elements in the drift chamber (DCH). Lower momentum tracks, which do not leave signals on many wires in the DCH due to the bending induced by the $1.5 \mathrm{~T}$ solenoid field, are reconstructed solely in the SVT. Charged hadron identification is performed by combining the measurements of the energy deposition in the SVT and in the $\mathrm{DCH}$ with the information from a Cherenkov detector (DIRC). Electrons are identified by the ratio of the energy deposited in the calorimeter (EMC) to the track momentum, the transverse profile of the shower, the energy loss in the DCH, and the Cherenkov angle in the DIRC. Muons are identified in the instrumented flux return (IFR), composed of resistive plate chambers and layers of iron.

We preselect a sample of hadronic events with at least four charged tracks. To reduce continuum background, we require that the ratio of the 2nd to the 0th order FoxWolfram [5] variables be less than 0.6. We then select a sample of partially reconstructed $B$ mesons in the channel $\bar{B}^{0} \rightarrow D^{*+}(X) \ell^{-} \bar{\nu}_{\ell}$, by retaining events containing a charged lepton $(\ell=e, \mu)$ and a low momentum pion (soft-pion, $\pi_{s}^{+}$) which may arise from the decay $D^{*+} \rightarrow$ $D^{0} \pi_{s}^{+}$. This sample of events is referred to as the "inclusive sample." The lepton momentum [6] must be in the range $1.4<p_{\ell^{-}}<2.3 \mathrm{GeV} / c$ and the soft-pion candidate must satisfy $60<p_{\pi_{s}^{+}}<190 \mathrm{MeV} / c$. The lepton and softpion minimum momenta are optimized to minimize un- certainties due to charm production in $B$ decays and tracking errors, respectively. Maximum momentum selections are determined by the available phase space. The two tracks must be consistent with originating from a common vertex, constrained to the beam-spot in the plane transverse to the beam axis. Then we combine $p_{\ell^{-}}, p_{\pi_{s}^{+}}$and the probability from the vertex fit into a likelihood ratio variable, optimized to reject $B \bar{B}$ background. Using conservation of momentum and energy, the invariant mass squared of the undetected neutrino is calculated as

$$
\mathcal{M}_{\nu}^{2} \equiv\left(E_{\text {beam }}-E_{D^{*}}-E_{\ell}\right)^{2}-\left(\vec{p}_{D^{*}}+\vec{p}_{\ell}\right)^{2},
$$

where $E_{\text {beam }}$ is half the total center-of-mass energy and $E_{\ell}\left(E_{D^{*}}\right)$ and $\vec{p}_{\ell}\left(\vec{p}_{D^{*}}\right)$ are the energy and momentum of the lepton (the $D^{*}$ meson). Since the magnitude of the $B$ meson momentum, $p_{B}$, is sufficiently small compared to $p_{\ell}$ and $p_{D^{*}}$, we set $p_{B}=0$ in obtaining Eq. (1). As a consequence of the limited phase space available in the $D^{*+}$ decay, the soft pion is emitted nearly at rest in the $D^{*+}$ rest frame. The $D^{*+}$ four-momentum can therefore be computed by approximating its direction as that of the soft pion, and parameterizing its momentum as a linear function of the soft-pion momentum. We select pairs of tracks with opposite electric charge for our signal $\left(\ell^{\mp} \pi_{s}^{ \pm}\right)$and same-charge pairs $\left(\ell^{ \pm} \pi_{s}^{ \pm}\right)$for background studies.

All events where $D^{*+}$ and $\ell^{-}$originate from the same $B$-meson, producing a peak near zero in the $\mathcal{M}_{\nu}^{2}$ distribution, are considered as signal candidates. Several processes contribute: (a) $\bar{B}^{0} \rightarrow D^{*+} \ell^{-} \bar{\nu}_{\ell}$ decays (primary); (b) $\bar{B} \rightarrow D^{*+}(n \pi) \ell^{-} \bar{\nu}_{\ell}$, where the $D^{*+}(n \pi)$ may or may not originate from an excited charm state $\left(D^{* * *}\right)$ and $n \geq 1$; (c) $\bar{B}^{0} \rightarrow D^{*+} \bar{D}, \bar{D} \rightarrow \ell^{-} X$ and $\bar{B}^{0} \rightarrow D^{*+} \tau^{-} \bar{\nu}_{\tau}, \tau^{-} \rightarrow$ $\ell^{-} \bar{\nu}_{\ell} \nu_{\tau}$ (cascade); (d) $\bar{B}^{0} \rightarrow D^{*+} h^{-}$(fake-lepton), where the hadron $(h=\pi, K)$ is erroneously identified as a lepton (in most of the cases, a muon). We also include radiative events, where photons with energy above $1 \mathrm{MeV}$ are emitted by any charged particle using PHOTOS v2.03 [7]. The signal region is $\mathcal{M}_{\nu}^{2}>-2 \mathrm{GeV}^{2} / c^{4}$ and the sideband is $-10<\mathcal{M}_{\nu}^{2}<-4 \mathrm{GeV}^{2} / c^{4}$.

The background in the inclusive sample consists of continuum and combinatorial $B \bar{B}$ events, which also include events where true $D^{*+}$ and $\ell^{-}$from the two different $B$ mesons are combined. We determine the number of signal events in our sample with a minimum $\chi^{2}$ fit to the $\mathcal{M}_{\nu}^{2}$ distribution in the interval $-10<\mathcal{M}_{\nu}^{2}<$ $2.5 \mathrm{GeV}^{2} / c^{4}$. We perform the fit in ten bins of the lepton momentum in order to reduce the sensitivity of the result to the details of the simulation. In each bin we fix the continuum contribution to the off-resonance events, rescaled to account for the luminosity ratio between the on- and the off-resonance samples, while we vary independently the number of signal events from primary, from $D^{* * *}$, and from combinatorial $B \bar{B}$, assuming the shapes predicted by the simulation. We fix the contributions from cascade and fake-lepton decays, which account for about $3 \%$ of the signal sample, to the Monte Carlo (MC) prediction. We fit 
eight different sets, divided by lepton kind and run condition. The reduced $\chi^{2} \mathrm{~s}$ range between 1.1 and 1.4. Figure 1(a) shows the result of the fit in the $\mathcal{M}_{\nu}^{2}$ projection. The number of signal events with $\mathcal{M}_{\nu}^{2}>-2 \mathrm{GeV}^{2} / c^{4}$ is $N^{\text {incl }}=(2170.64 \pm 3.04($ stat $) \pm 18.1$ (syst) $) \times 10^{3} . \quad$ The statistical uncertainty includes the statistical uncertainties of the off-resonance and of the simulated events.

We then reconstruct for $D^{0} \rightarrow K^{-} \pi^{+}$decays in the inclusive sample. We consider all tracks in the event, aside from the $\ell^{-}$and $\pi_{s}^{+}$, with momenta in the direction transverse to the beam axis exceeding $0.2 \mathrm{GeV} / c$. We combine pairs of tracks with opposite charge, and compute the invariant mass $M_{K \pi}$ assigning the kaon mass to the track with charge opposite the $\pi_{s}$ charge. The kaon candidate must satisfy a loose kaon identification criterion that retains more than $80 \%$ of true kaons, while rejecting more than $95 \%$ of pions. We select events in the mass range $1.82<M_{K \pi}<1.91 \mathrm{GeV} / c^{2}$. We combine each $D^{0}$ candidate with the $\pi_{s}^{+}$and compute the mass difference $\Delta M=$ $M\left(K^{-} \pi^{+} \pi_{s}^{+}\right)-M\left(K^{-} \pi^{+}\right)$. We look for signal in the range of $142.4<\Delta M<149.9 \mathrm{MeV} / c^{2}$.

This exclusive sample consists of signal events and of the following background sources: continuum, combinatorial $B \bar{B}$, uncorrelated peaking $D^{*+}$, and Cabibbosuppressed decays. We subtract the continuum background using rescaled off-resonance events selected with the same criteria as the on-resonance data. Combinatorial events are due to any combination of three tracks, in which at least one does not come from the $D^{*+}$. We determine their number from simulated $B \bar{B}$ events. We normalize the simulated events to the data in the $\Delta M$ sideband, $153.5<$ $\Delta M<162.5 \mathrm{MeV} / c^{2}$, properly accounting for the small fraction of signal events (less than 1\%) contained in the

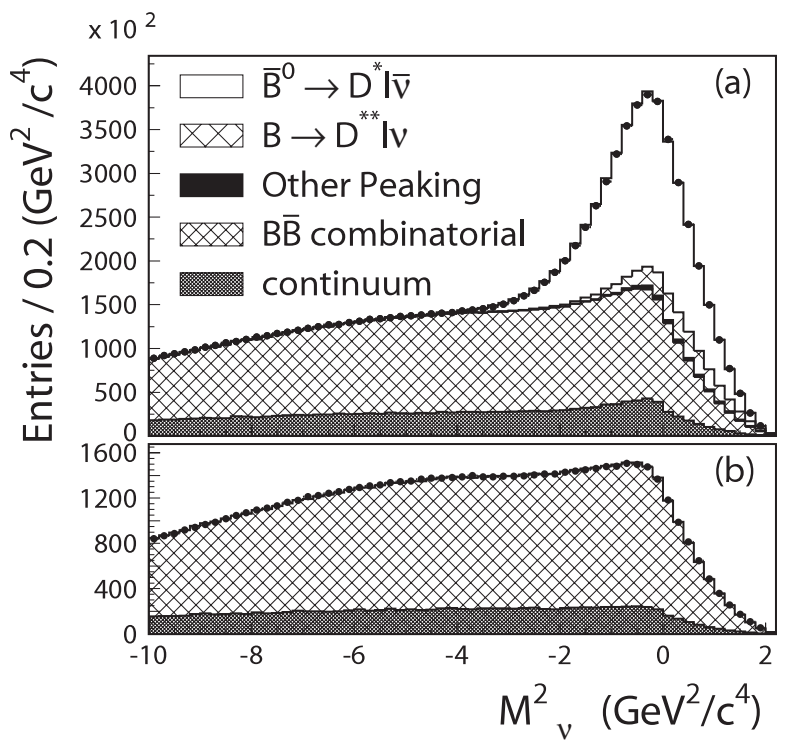

FIG. 1. The $\mathcal{M}_{\nu}^{2}$ distribution of the inclusive sample, for rightcharge (a) and wrong-charge (b) samples. The data are represented by solid points with uncertainty. The MC fit results are overlaid to the data, as explained in the figure. sideband. We verify that the background shape is properly described in the simulation using a sample of $D^{*+}$-depleted events, obtained as follows. We use wrong-charge events where the kaon has the same charge as the $\pi_{s}$, selected in the $\mathcal{M}_{\nu}^{2}$ sideband. More than $95 \%$ of the events so selected in the $\Delta M$ signal region are combinatorial background, with a residual peaking component from Cabibbosuppressed decays $\left(K^{+} K^{-}\right.$and $\pi^{+} \pi^{-}$, see below). After normalizing the level of the simulated events in the sideband, the number of events in the signal region is consistent with the data within the statistical precision of $\pm 1.3 \%$.

The background from uncorrelated peaking $D^{*+}$ decays occurs when the $D^{*+}$ and the $\ell^{-}$originate from the two different $B$ mesons. These events exhibit a peak in $\Delta M$ but behave as combinatorial background in $\mathcal{M}_{\nu}^{2}$. We compute their number in the $\mathcal{M}_{\nu}^{2}$ sideband data and rescale it to the $\mathcal{M}_{\nu}^{2}$ signal region using the $\mathcal{M}_{\nu}^{2}$ distribution of the combinatorial simulated events.

Cabibbo-suppressed decays $D^{0} \rightarrow K^{-} K^{+}\left(\pi^{-} \pi^{+}\right)$contribute to the peaking background, where one of the kaons (pions) is wrongly identified as a pion (kaon). Simulation shows that these events peak in $\Delta M$, while they exhibit a broad $M_{K \pi}$ distribution. We subtract this background source using the simulation prediction. It should be noted that the contribution from doubly Cabibbo-suppressed decays is negligible. Figure 2 shows the continuumsubtracted distribution for the data with the simulated $B \bar{B}$ backgrounds overlaid.

The exclusive selection yields $N^{\text {excl }}=(3.381 \pm$ $0.029) \times 10^{4}$ signal events, where the uncertainty is statistical only. The detailed composition of the inclusive and exclusive data sets is listed in Table I.

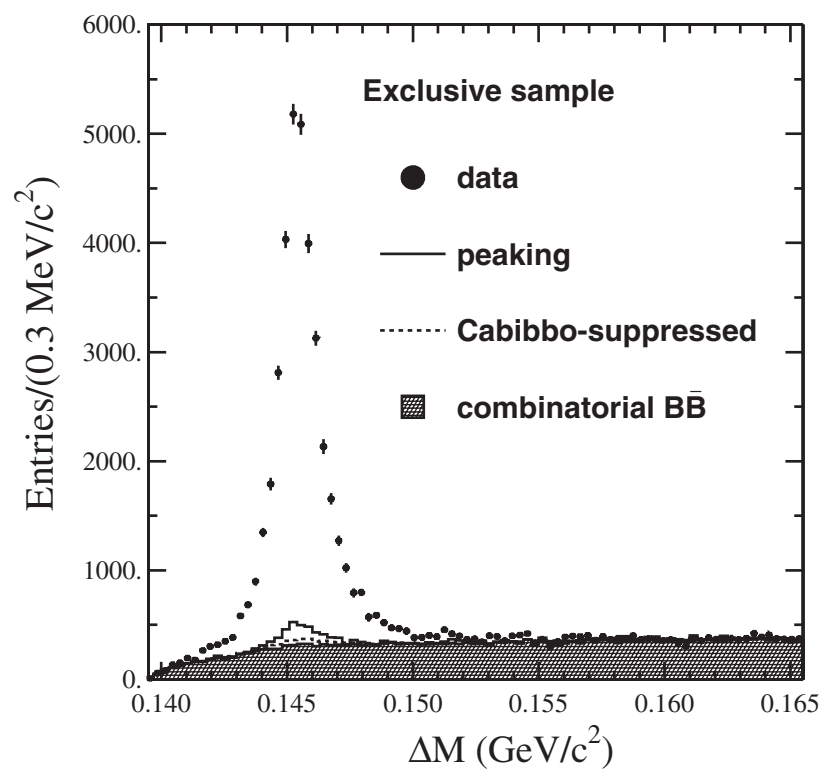

FIG. 2. Continuum-subtracted $\Delta M$ distribution for data (points with error bars) and backgrounds overlaid as explained in the figure. 
TABLE I. The inclusive and exclusive samples.

\begin{tabular}{lcc}
\hline \hline \multicolumn{1}{c}{ Source } & Inclusive $\left(\times 10^{6}\right)$ & Exclusive $\left(\times 10^{4}\right)$ \\
\hline Data & $4.4124 \pm 0.0021$ & $4.727 \pm 0.022$ \\
Continuum & $0.46 \pm 0.0021$ & $0.309 \pm 0.017$ \\
Combinatorial $B \bar{B}$ & $1.7817 \pm 0.0007$ & $0.819 \pm 0.005$ \\
Peaking & - & $0.163 \pm 0.008$ \\
Cabibbo-suppressed & - & $0.055 \pm 0.001$ \\
Signal & $2.1706 \pm 0.0030$ & $3.381 \pm 0.029$ \\
\hline \hline
\end{tabular}

We compute the branching fraction as

$$
\mathcal{B}\left(D^{0} \rightarrow K^{-} \pi^{+}\right)=N^{\mathrm{excl}} /\left(N^{\mathrm{incl}} \zeta \varepsilon_{\left(K^{-} \pi^{+}\right)}\right)
$$

where $\varepsilon_{\left(K^{-} \pi^{+}\right)}=(36.96 \pm 0.09) \%$ is the $D^{0}$ reconstruction efficiency from simulation, and $\zeta=1.033 \pm 0.002$ is the selection bias introduced by the partial reconstruction. Only the statistical uncertainties are reported here. The bias factor $\zeta$ accounts for the larger efficiency of the inclusive event reconstruction for final states with two or fewer tracks from $D^{0}$ decays due to the smaller density of hits near the $\pi_{s}^{+}$track. We study these effects by comparing data and simulated distributions of the number of charged tracks in each event $\left(n_{\text {trk }}\right)$ and of other quantities sensitive to the soft-pion isolation (angle to nearest track and track density within $10^{\circ}$ cone around the $\pi_{s}^{+}$direction). We weight simulated events to reproduce the data and recompute the bias. We observe an efficiency variation of $0.33 \%$ due to $n_{\text {trk }}$ and $0.08 \%$ due to the other variables.

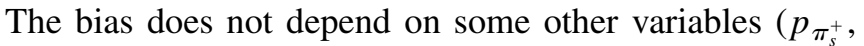
number of $\pi_{s}^{+}$hits in the SVT). The systematic uncertainty due to this selection is $\pm 0.35 \%$.

The main systematic uncertainty on $N^{\text {incl }}$ is due to the nonpeaking combinatorial $B \bar{B}$ background. We perform the same fit to the $\ell^{ \pm} \pi_{s}^{ \pm}$background control sample and the signal-dominated sample. We take the systematic uncertainty in the combinatorial background to be the rms scatter in the ratio, calculated for each $\mathcal{M}_{\nu}^{2}$ bin as shown in Fig. 1(b), of continuum-subtracted data to the value of the combinatorial background determined from the fit, resulting in an uncertainty of $0.89 \%$. As first noticed in [8], the decays $\bar{B}^{0} \rightarrow \ell^{-} \bar{\nu}_{\ell} D^{+}$, with $D^{+} \rightarrow K^{*} \rho(\omega) \pi^{+}$, constitute a right-charge peaking background, because the charged pion is produced almost at rest in the $D^{+}$rest frame. In order to estimate the systematic uncertainty due to this peaking combinatorial background, we vary its total fraction by $\pm 100 \%$ in the $B \bar{B}$ events in the MC calculations. The corresponding systematic uncertainty is $\pm 0.34 \%$.

We consider systematic uncertainties affecting the signal $\mathcal{M}_{\nu}^{2}$ distribution. Final state photon radiation in $D^{0}$ decays alters the distribution of $M_{K^{-}} \pi^{+}$and thus affects the efficiency computation. We estimate a systematic uncertainty of $\pm 0.50 \%$ due to the final state photon radiation by varying by $\pm 30 \%$ the fraction of reconstructed events in the simulation where at least one photon above $1 \mathrm{MeV}$ is emitted in the $D^{0} \rightarrow K^{-} \pi^{+}$decay.

We also vary, by $\pm 30 \%$, the fractions of cascade and fake-lepton decays, which are not determined by the fit. Finally, we vary in turn by $\pm 100 \%$ the number of events from each of the five sources constituting the $D^{* *}$ samples (two narrow and two broad resonant states, and nonresonant $D^{*+} \pi$ combinations; these last are described using the model of ref. [9]). We repeat the measurement and take the variation as the systematic uncertainty.

The dominant contribution to the systematic uncertainty on $N^{\text {excl }}$ is due to the charged-track reconstruction efficiency. The single charged-track reconstruction efficiency is determined with $0.50 \%$ precision, which corresponds to $\pm 1.00 \%$ overall uncertainty. The efficiency for $K^{-}$identification is measured with $\pm 0.70 \%$ systematic uncertainty from a large sample of $D^{*+} \rightarrow D^{0} \pi_{s}^{+}, D^{0} \rightarrow K^{-} \pi^{+}$decays, produced in $e^{+} e^{-} \rightarrow c \bar{c}$ events. To estimate the systematic uncertainty due to the combinatoric background subtraction on $N^{\text {excl }}$, we first vary the number of events from combinatorial background below the signal peak by $\pm 1.3 \%$, corresponding to the statistical uncertainty obtained from the control sample described above. This translates in $\pm 0.3 \%$ systematic uncertainty on the result. We vary the number of signal events contained in the sideband by $\pm 30 \%$ for background normalization. This induces a systematic uncertainty of $\pm 0.16 \%$. We vary the fraction of events from Cabibbo-suppressed decays by $\pm 10 \%$. The systematic uncertainty due to the uncorrelated peaking (from data) is negligible.

When comparing the simulated $M_{K \pi}$ distribution to the data in a high purity signal sample (obtained by asking, in addition to the other cuts, that the hard pion

TABLE II. The systematic uncertainties of $\mathcal{B}\left(D^{0} \rightarrow K^{-} \pi^{+}\right)$.

\begin{tabular}{llc}
\hline \hline \multicolumn{1}{c}{ Source } & $\delta(\mathcal{B}) / \mathcal{B}(\%)$ \\
\hline \multirow{2}{*}{$N^{\text {incl }}$} & Selection bias & \pm 0.35 \\
& Nonpeaking combinatorial background & \pm 0.89 \\
& Peaking combinatorial background & \pm 0.34 \\
Soft-pion decays in flight & \pm 0.10 \\
Fake leptons & \pm 0.08 \\
Cascade decays & \pm 0.08 \\
Monte Carlo events shape & \pm 0.08 \\
Continuum background & \pm 0.05 \\
$D^{* *}$ production & \pm 0.02 \\
& Photon radiation & \pm 0.02 \\
$N^{\text {excl }}$ & Tracking efficiency & \pm 1.00 \\
$K^{-}$identification & \pm 0.70 \\
$D^{0}$ invariant mass & \pm 0.56 \\
Photon radiation in $D^{0}$ decay & \pm 0.50 \\
Combinatorial background shape & \pm 0.30 \\
Combinatorial background normalization & \pm 0.16 \\
Soft-pion decay & \pm 0.12 \\
Cabibbo-suppressed decays & \pm 0.10 \\
\hline \hline
\end{tabular}


fails $K, p$ and $\ell$ identification criteria and $0.1435<\Delta M<$ $0.1475 \mathrm{GeV} / c^{2}$ ) we observe a slight discrepancy, causing $\pm 0.56 \%$ systematic uncertainty in the reconstruction efficiency. We compute the total relative systematic uncertainty of $\pm 1.80 \%$ from the quadratic sum of all uncertainties described above and listed in Table II. We cross check our results using different definitions of the $\Delta M$ and $M_{K^{-}} \pi^{+}$signal regions and particle identification. We split our data into different subsamples, depending on the run conditions. All the results are consistent.

In summary, we have measured the absolute branching fraction of $D^{0} \rightarrow K^{-} \pi^{+}$decay with partial reconstruction of $\bar{B}^{0} \rightarrow D^{*+}(X) \ell^{-} \bar{\nu}_{\ell}$, and obtain the result

$$
\mathcal{B}\left(D^{0} \rightarrow K^{-} \pi^{+}\right)=(4.007 \pm 0.037 \pm 0.072) \%,
$$

where the first uncertainty is statistical and the second uncertainty is systematic. This result is comparable in precision with the present world average, and it is consistent with it within 2 standard deviations.

We are grateful for the excellent luminosity and machine conditions provided by our PEP-II colleagues, and for the substantial dedicated effort from the computing organizations that support BABAR. The collaborating institutions wish to thank SLAC for its support and kind hospitality. This work is supported by DOE and NSF (USA), NSERC (Canada), CEA and CNRS-IN2P3 (France), BMBF and DFG (Germany), INFN (Italy), FOM (The Netherlands),
NFR (Norway), MIST (Russia), MEC (Spain), and PPARC (United Kingdom). Individuals have received support from the Marie Curie EIF (European Union) and the A. P. Sloan Foundation.

*Deceased

${ }^{\dagger}$ Also with Università di Perugia, Dipartimento di Fisica, Perugia, Italy.

${ }^{*}$ Also with Università della Basilicata, Potenza, Italy.

${ }^{\S}$ Also with IPPP, Physics Department, Durham University, Durham DH1 3LE, United Kingdom.

[1] The inclusion of charge-conjugate reactions is implied throughout this Letter.

[2] Q. He et al. (CLEO-c Collaboration), Phys. Rev. Lett. 95, 121801 (2005); 96, 199903(E) (2006).

[3] W.-M. Yao et al. (Particle Data Group), J. Phys. G 33, 1 (2006).

[4] B. Aubert et al. (BABAR Collaboration), Nucl. Instrum. Methods Phys. Res., Sect. A 479, 1 (2002).

[5] G. C. Fox and S. Wolfram, Phys. Rev. Lett. 41, 1581 (1978).

[6] Four-momenta are computed in the $Y(4 S)$ rest frame.

[7] E. Barberio and Z. Was, Comput. Phys. Commun. 79, 291 (1994).

[8] M. Artuso et al. (CLEO Collaboration), Phys. Rev. Lett. 80, 3193 (1998).

[9] J. L. Goity and W. Roberts, Phys. Rev. D 51, 3459 (1995). 